\title{
A Survey on Quality of Service in the Voice Over IP Technology
}

\author{
Homero Toral-Cruz \\ University of Quintana Roo, Mexico \\ José Luis Vázquez-Ávila \\ Rafael Sánchez-Lara \\ Universidad Autónoma del Carmen, Mexico \\ Joel Antonio Trejo-Sánchez \\ CONACyT - Centro de Investigación en Matemáticas, Mexico \\ J.A. Alvarez-Chavez \\ Centro de Investigacion e Innovacion Tecnologica - IPN, Mexico
}

Doi: 10.19044/esj.2018.c5p15 ～URL:http://dx.doi.org/10.19044/esj.2018.c5p15

\begin{abstract}
Voice services can be transmitted by circuit switched and packet switched networks (Internet). Voice over Internet Protocol (VoIP) is one of the most attractive and important service in telecommunication networks, current implementations of VoIP have two main types of architectures, which are based on H.323 and Session Initiation Protocol (SIP). However, when the voice traffic is transported over Internet, the packet based transmission may introduce impairments and it has influence on the Quality of Service (QoS) perceived by the end users. The voice quality of VoIP systems depends on many QoS parameters. Particularly, One Way Delay (OWD), jitter and Packet Loss Rate (PLR) have an important impact on voice quality. This survey presents the main concepts relating to the VoIP technology and quality of service issues.
\end{abstract}

Keywords: Voice over Internet Protocol, Quality of Service, QoS parameters

\section{Introduction}

In the last decades, voice transmission has become as one of the most attractive and important service in the telecommunications networks. Voice services can be transmitted by circuit switched and packet switched networks (Toral et al., 2013). The most common examples of circuit switched and packet switched networks are the Public Switched Telephone Network 
(PSTN) and Internet, respectively (Kurose and Ross, 2005). Compared to traditional resource-dedicated PSTN, Internet is resource shared. Therefore, the conditions in the PSTN are totally different from those in the Internet, and this fact has influence on the voice quality (Toral et al., 2013, Toral et al., 2008).

VoIP is the real-time transmission of voice between two or more parties by using IP technologies, poised to replace the circuit switched telephony service in the future and carry voice packets transparently through the Internet (Toral et al., 2012, Toral et al., 2013). Current implementations of VoIP have two main types of architectures, which are based on H.323 ratified by International Telecommunication Union (ITU-T) (ITU-T Recommendation H.323, 2007) and SIP developed by working group of Internet Engineering Task Force (IETF) (Rosenberg, 2007). The above mentioned architectures do not provide QoS and they consist of three main logical components: terminal, signaling server, and GW (Toral et al., 2011, Toral et al., 2013).

A basic VoIP system consists mainly of three parts, the source terminal (sender), destination terminal (receiver) and the IP network (Internet) (Toral et al., 2011, Toral et al., 2013). The IP network is characterized by its random and complex nature, as result of the convergence of information and media transmission (voice, video and data) through the same communication channel. As there are a very high (and increasing) number of nodes (i.e., devices) connected to the network, and these are being added in a random, decentralized manner, the network is asymmetric and also practically random in both its topology and its usage. Additionally, the service provided by the Internet is generally a "best effort" type (Park, 2005), which means that the nodes, with some exceptions, do not differentiate between traffic types and there is neither resource reservation nor prioritization (Estrada et al., 2009-1, Estrada et al., 2009-2).

Congestion, due to the high demand of network resources, is a cause of the impairment of the quality of service, which consists of delay problems (i.e., the OWD and its variation, namely jitter) and PLR. For time-critical communications, such as VoIP, the above impairments can have high impact on the QoS (Toral et al., 2011-1, Estrada et al., 2009-1).

\section{Telecommunication Networks}

In telecommunications networks there are many networks, in this survey two of them are studied, circuit switched and packet switched networks. 


\section{Circuit Switched Network}

The communication via circuit switched networks implies that there is a dedicated communication path between two or more terminals all through the communication session. Therefore, the resources (links and nodes) are reserved exclusively for information exchanges between source and destination terminals (Toral et al., 2011, Toral et al., 2013).

In this switching technology, the nodes do not examine the contents of the information transmitted; the decision on where to send the information received is made just once at the beginning of connection and remains during the connection. Thus, the delay introduced by a node is almost negligible. After the circuit has been established, the transmission delay is small and is kept constant through the duration of the connection (Toral et al., 2011, Toral et al., 2013). Therefore, in this network technology, the voice quality is guaranteed, while the bandwidth utilization is not efficient.

The most common examples of circuit switched network are the PSTN and Integrated Services Digital Network (ISDN) (Toral et al., 2011, Toral et al., 2013).

\section{Packet Switched Network}

In the packet switched networks, the information is split up by the source terminal into blocks of moderate size, called packets. These packets can be autonomous, i.e., they are capable of moving on the network thanks to a header that contains the source and destination addresses (Fiche and Hébuterne, 2004).

The packet is sent to the first node (router), the router receives the packet, and it examines the header and forwards the packet to the next appropriate router. This technique of inspection and retransmission is called "store-and-forward", and it is accomplished in all routers of the path until the packet reaches its destination, unless the packet is lost. After reaching the destination, the destination terminal strips off the header of the packet to obtain the actual data that was originated at the source (Toral et al., 2011, Toral et al., 2013).

In this switching technology, the source sends packets and the network multiplexes the packets from various origins in the same resources to optimize their use. In this way several communications can share the same resources (Toral et al., 2011, Toral et al., 2013).

The packet switching enables a better use of the bandwidth than circuit switching because the resources are shared. However, the multiplexing of different connections on the same resources causes delays and packet losses, which do not happen with circuit switched technology (Kurose and Ross, 2001). In this network technology, the voice quality is not 
guaranteed due to its shared nature, thus VoIP is susceptible to suffer impairments, which result in voice quality degradation (Jo et al., 2002).

\section{VoIP Architectures and Protocols}

VoIP consists of a set of architectures and protocols for managing the transmission of voice packets through IP network, the most important are explained below.

\section{H.323 Architecture}

H.323 is a set of protocols for voice, video, and data conferencing over packet switched networks, such as Internet, that do not provide a guaranteed QoS (ITU-T Recommendation H.323, 2007). The ITU-T H.323 recommendation describes the components of $\mathrm{H} .323$ architecture, as can be seen in Figure 1. An H.323 terminal is an endpoint on the network, which provides real-time, two-way communications with another H.323 terminal, gateway, or multipoint control unit (MCU). The gateway is an H.323 entity on the network, which allows intercommunication between IP networks and legacy circuit-switched networks, such as ISDN and PSTN. The gatekeeper is an H.323 entity on the network, which performs the role of the central manager of VoIP services to the endpoints. The MCU is an H.323 entity on the network, which provides the capability for three or more terminals and a gateway to participate in a multipoint conference.

The H.323 architecture is partitioned into zones. Each zone is composed of the collection of all terminals, a gateway, and MCU managed by a single gatekeeper.

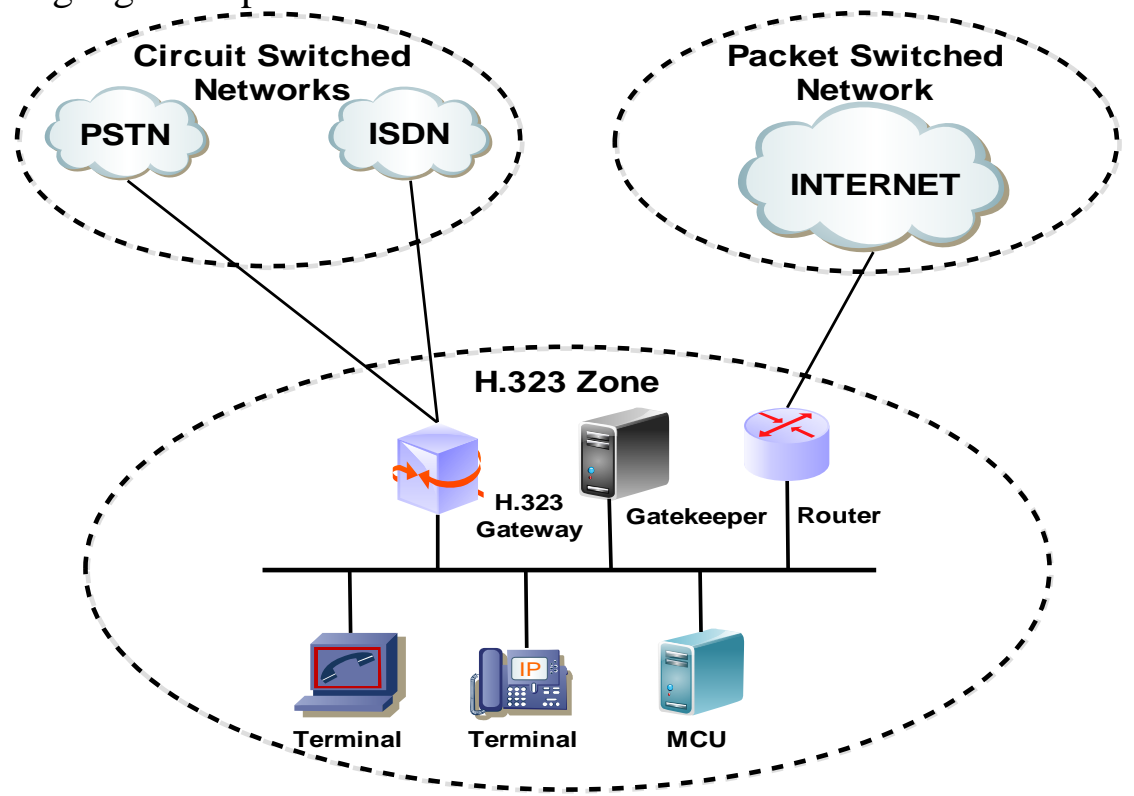

Figure 1: H.323 architecture 


\section{SIP Architecture}

SIP is an application layer control protocol that can establish, modify, and terminate multimedia sessions or calls. SIP was developed by IETF in reaction to the ITU-T H.323 recommendation (Rosenberg, 2007).

The two major components in a SIP network are the user agent (UA) and network servers (registrar server, location server, proxy server, and redirect server), as shown in Figure 2. The user Agent is an application that interacts with the user and contains both a user agent client (UAC) and user agent server (UAS). A user agent client initiates SIP requests, and a user agent server receives SIP requests and return responses on user behalf. The registrar server is a SIP server that accepts only registration requests issued by user agents for the purpose of updating a location database with the contact information of the user specified in the request. The proxy server is an intermediary entity that acts both as a server to user agents by forwarding SIP requests and as a client to other SIP servers by submitting the forwarded requests to them on behalf of user agents or proxy servers. The redirect server is a SIP server that helps to locate UAs by providing alternative locations where the user can be reachable, i.e., provides address mapping services. It responds to a SIP request destined to an address with a list of new addresses. A redirect server doesn't accept calls, doesn't forward requests, and doesn't it initiate any of its own.

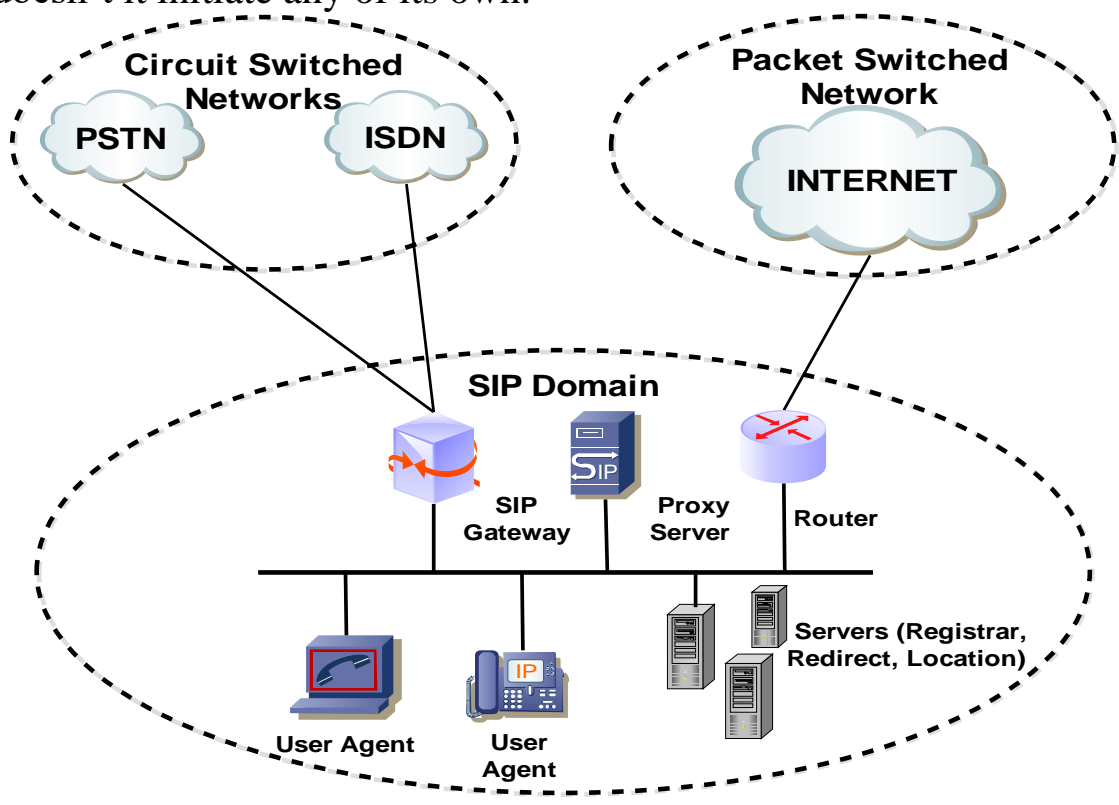

Figure 2: SIP architecture 


\section{QoS Parameters}

Several parameters influencing voice quality on IP networks, in particular OWD, jitter and PLR are the most critical (Sherif and Crossman, 1995).

\section{OWD}

The delay experienced by a packet across a path consists of several components: propagation, processing, transmission, and queuing delays. The Internet metric one way delay (ITU-T Recommendation G.114, 2003) is the time needed for a packet to traverse the network from a source to a destination host.

\section{Jitter}

When packets are transmitted from a source to a destination over IP networks, packets may experience variable delay, called delay jitter. The Inter-Arrival Time (IAT) on the receiver side is not constant even if the packet Inter-Departure Time (IDT) on the sender side is constant. As a result, packets arrive at the destination with varying delays (between packets) referred to as jitter.

\section{PLR}

There are two main transport protocols used on IP networks: User Datagram Protocol (UDP) and Transmission Control Protocol (TCP). While UDP protocol does not allow any recovery of transmission errors, TCP include an error recovery process. However, the voice transmission over TCP connections is not very realistic. This is due to the requirement for realtime (or near real-time) operations in most voice related applications. As a result, the choice is limited to the use of UDP which involves packet loss problems.

Amongst the different quality elements, packet loss is the main impairment which makes the VoIP perceptually most different from the public switched telephone network. Packet loss can occur in the network or at the receiver side, for example, due to excessive network delay in case of network congestion.

\section{Conclusion}

In telecommunications networks there are many networks, in this survey two of them are studied, circuit switched and packet switched networks. The most common examples of circuit switched and packet switched networks are the PSTN and Internet, respectively. In mid 1990's, the PSTN and Internet started to converge. As result of this merging, appears the convergence of voice and data networks. However, with this 
convergence, a new technical challenge has emerged. The converged network (Internet) is based on the best effort service and it does not guarantee a quality of services level to meet requirements of real time applications, such as VoIP. VoIP is one of the most sensitive services of QoS and to compete with traditional service demands specific QoS levels. Current implementations of VoIP have two main types of architectures, which are based on H.323 and SIP. The above mentioned architectures do not provide QoS. Several parameters influencing voice quality on VoIP, in particular OWD, jitter and PLR are the most critical. The QoS is an important subject that takes a central place in the IP network technologies, it is a complex subject and its analysis involves mathematical disciplines such as: probability theory and stochastic processes.

\section{References:}

1. J.F. Kurose and K.W. Ross, Computer networking, Addison-Wesley Boston, USA, 2001.

2. K.I. Park, QOS in packet networks, Springer Science + Business Media, Inc, Boston, MA, 2005.

3. J. Jo, G. Hwang and H. Yang, "Characteristics of QoS Parameters for VoIP in the Short-Haul Internet," Proc. International Conferences on Info-tech and Info-net (ICII), IEEE, Beijing, China, 29 October - 01 November, 2001, pp. 498-502.

4. M.H. Sherif and A. Crossman, "Overview of speech packetization," Proc. IEEE Symposium on Computers and Communications (ISCC), IEEE, Alexandria, Egypt, 27-29 July, 1995, pp. 296-304.

5. Homero Toral-Cruz, Al-Sakib Khan Pathan, and Julio C. Ramírez Pacheco. Accurate Modeling of VoIP Traffic QoS Parameters in Current and Future Networks with Multifractal and Markov Models. Mathematical and Computer Modelling Journal, 57 (11-12) (2013) 2832-2845.

6. Homero Toral-Cruz, D. Torres, C. Hernandez, and L. Estrada. SelfSimilarity, Packet Loss, Jitter, and Packet Size: Empirical Relationships for VoIP. In Proceeding of the 18th International Conference on Electronics, Communications and Computers (CONIELECOMP 2008). Cholula, Puebla, 2008, pp. 11-16.

7. Homero Toral-Cruz, Julio Cesar Ramirez-Pacheco, Pablo VelardeAlvarado, Al-Sakib Khan Pathan. VoIP in Next Generation Converged Networks. Building Next-Generation Converged Networks: Theory and Practice. CRC Press, Taylor \& Francis Group, USA, 2013, pp. 337-360.

8. Homero Toral-Cruz, D. Torres, L. Estrada, Analysis and Modeling of QoS Parameters in VoIP Traffic. Advancements in Distributed 
Computing and Internet Technologies: Trends and Issues. IGI Global Publishers, USA, 2012, pp. 1-22.

9. ITU-T Recommendation H.323. Packet-Based Multimedia Communications Systems. International Telecommunications Union. Geneva, Switzerland, 2007.

10. J. Rosenberg, et al. SIP: Session Initiation Protocol (RFC 3261). Internet Engineering Task Force, 2002.

11. Homero Toral-Cruz, J. Argaez, L. Estrada, D. Torres. An Introduction to VoIP: End-to-End Elements and QoS Parameters. VoIP Technologies. INTECH Publisher, Croatia, 2011, pp. 79-94.

12. L. Estrada, D. Torres, and Homero Toral-Cruz. Analytical Investigation of the Performance of Packet-level FEC Techniques in VoIP Communications. Proceeding of the 9th WSEAS International Conference on Signal, Speech and Image Processing (SSIP). 9th WSEAS International Conference on Multimedia, Internet \& Video Technologies (MIV). Budapest, Hungary, 2009, pp. 192-197.

13. L. Estrada, D. Torres, Homero Toral-Cruz. Analytical Description of a Parameter-based Optimization of the Quality of Service for VoIP Communications. WSEAS Transactions on Communications, 9 (8) (2009) 1042-1052.

14. G. Fiche and G. Hébuterne. Communicating systems \& networks: traffic \& Performance. Kogan Page Science, London and Sterling, VA, 2004.

15. J.F. Kurose and K.W. Ross. Computer networking. Addison-Wesley Boston, USA, 2001.

16. ITU-T Recommendation G.114. One way transmission time, International Telecommunications Union. Geneva, Switzerland, 2003. 